\title{
FORMULAE FOR THE RELATIVE CLASS NUMBER OF AN IMAGINARY ABELIAN FIELD IN THE FORM OF A DETERMINANT
}

\author{
RADAN KUČERA
}

\begin{abstract}
There is in the literature a lot of determinant formulae involving the relative class number of an imaginary abelian field. Usually such a formula contains a factor which is equal to zero for many fields and so it gives no information about the class number of these fields. The aim of this paper is to show a way of obtaining most of these formulae in a unique fashion, namely by means of the Stickelberger ideal. Moreover some new and non-vanishing formulae are derived by a modification of Ramachandra's construction of independent cyclotomic units.
\end{abstract}

\section{$\S 1$. Introduction}

In [2], Carlitz and Olson computed Maillet's determinant for an odd prime $p$. For any positive integer $m$ and any integers $a, b$ relatively prime to $m$ let $R_{m}\left(a b^{-1}\right)$ means the integer determined by $R_{m}\left(a b^{-1}\right) b \equiv a(\bmod m)$ and $0<R_{m}\left(a b^{-1}\right) \leq m$. Carlitz and Olson obtained the following formula

$$
\begin{aligned}
\operatorname{det} & \left(R_{p}\left(a b^{-1}\right)-\frac{p}{2}\right)_{a, b \in\{1, \ldots,(p-1) / 2\}} \\
= & -\frac{1}{2} \cdot \operatorname{det}\left(R_{p}\left(a b^{-1}\right)\right)_{a, b \in\{1, \ldots,(p-1) / 2\}} \\
= & \pm \frac{1}{2} p^{(p-3) / 2} \cdot h_{K_{p}}^{-},
\end{aligned}
$$

where $h_{K_{p}}^{-}$means the relative class number of the $p$-th cyclotomic field $K_{p}$.

This result was generalized by Metsänkylä in [18] to any prime power cyclotomic field (see also [15] which together with [24] shows the relationship with the Stickelberger ideal) and by Tateyama in [27] to any cyclotomic field. For a positive integer $m \not \equiv 2(\bmod 4), m \neq 1$, let $n$ and $n^{+}$denote the

Received June 10, 1998.

1991 Mathematics Subject Classification: 11R29; 11R20.

This research is supported by the grant 201/97/0433 of the Grant Agency of the Czech Republic. 
number of prime ideals dividing $m$ in the $m$-th cyclotomic field $K_{m}$ and in its maximal real subfield $K_{m}^{+}$, respectively. Tateyama proved that

$$
\operatorname{det}\left(R_{m}\left(a b^{-1}\right)\right)_{a, b \in C}= \begin{cases}(-m)^{\left[K_{m}^{+}: \mathbb{Q}\right]-1} 2^{n-1} \cdot h_{K_{m}}^{-} & \text {if } n=n^{+} \\ 0 & \text { otherwise }\end{cases}
$$

where $C$ is the set of all positive integers less than $m / 2$ which are relatively prime to $m$. The same result was obtained independently by Wang (see [29]) whose formula contains the class number only implicitly.

An interesting paper on Maillet's determinant was also written by Okada ([19]) who observed the connection between Maillet's determinant (1) and the Stickelberger ideal of the $p$-th cyclotomic field. He also generalized Maillet's determinant for an odd prime $p$ in a different direction: taking any system of representatives $C$ of $(\mathbb{Z} / p \mathbb{Z})^{\times} /\{1,-1\}$ he proved that

$$
\operatorname{det}\left(R_{p}\left(r s^{-1}\right)\right)_{r, s \in C}=-\frac{1}{2}(-p)^{(p-3) / 2}\left(1+A_{C}\right) h_{K_{p}}^{-}
$$

Here

$$
A_{C}=\frac{2}{p-1} \sum_{\chi \in X^{-}} c_{\chi} c_{\bar{\chi}} B_{1, \chi}^{-1}
$$

where the sum is taken over the set of all odd Dirichlet characters modulo $p, B_{1, \chi}$ means the first generalized Bernoulli number, and $c_{\chi}=\sum_{a \in C} \chi(a)$. Comparing with (2) we obtain $A_{\{1, \ldots,(p-1) / 2\}}=-3$, which follows also directly from $\left[19\right.$, Proposition $\left.^{1}\right]$.

A connection between Maillet's determinant and the Stickelberger ideal of an odd prime power cyclotomic field was studied by Fuchs in [5].

A generalization of the Maillet's determinant to any imaginary abelian field $K$ was introduced by Girstmair (see [8]). It is easy to see that his result can be equivalently stated as follows. Let $m$ be the conductor of $K$; for any automorphism $\sigma \in \operatorname{Gal}(K / \mathbb{Q})$ put

$$
E_{\sigma}=m \sum_{\substack{j \bmod ^{\times} m \\(j, K)=\sigma}}\left(2\left\langle\frac{j}{m}\right\rangle-1\right),
$$

\footnotetext{
${ }^{1}$ Let us mention that this proposition works not only for $\theta^{\prime}=\sum \frac{R(\sigma)}{p} \sigma^{-1}$ (in Okada's notation), but also for $\theta^{*}=\sum \frac{R(\sigma)}{p} \sigma$ and that the identity [19, p. 169, row 4] should be read as $s\left(S_{0}\right)=\left(\sigma_{-1}+\sigma_{(p+1) / 2}-1\right) \theta^{*}$. See also Proposition 2 in Section 5 of this text.
} 
where $\langle x\rangle$ means the fractional part of a rational number $x$ (i.e., $0 \leq\langle x\rangle<1$, $x-\langle x\rangle \in \mathbb{Z}$ ) and $j$ in the sum runs over all those elements of a reduced residue system modulo $m$ which satisfy $(j, K)=\sigma$, i.e., $\sigma$ equals to the restriction to $K$ of the automorphism of $K_{m}$ which sends each root of unity to its $j$-th power. Let $C$ be a system of representatives of $\operatorname{Gal}(K / \mathbb{Q}) / \operatorname{Gal}\left(K / K^{+}\right)$. Then Girstmair's result is

$$
\operatorname{det}\left(E_{\sigma \tau^{-1}}\right)_{\sigma, \tau \in C}=(-2 m)^{\left[K^{+}: \mathbb{Q}\right]} \cdot q_{K} \cdot \frac{h_{K}^{-}}{Q_{K} w_{K}},
$$

where $h_{K}^{-}, Q_{K}$, and $w_{K}$ are the relative class number of $K$, the Hasse unit index of $K$, and the number of roots of unity in $K$, respectively, $\left[K^{+}: \mathbb{Q}\right]$ is the degree of the maximal real subfield $K^{+}$of $K$, and $q_{K}$ can be easily described by means of odd Dirichlet characters corresponding to $K$ (see Notation).

Maillet's determinant was modified by Endô (see [4]), who for an odd prime $p$ introduced the matrix $\left(R_{p}^{\prime}\left(a b^{-1}\right)\right)_{a, b \in\{1, \ldots,(p-1) / 2\}}$, where $R_{p}^{\prime}\left(a b^{-1}\right)$ means the integer satisfying $R_{p}^{\prime}\left(a b^{-1}\right) b \equiv a(\bmod p)$ and $-p / 2<R_{p}^{\prime}\left(a b^{-1}\right)$ $<p / 2$. Endô computed determinants of products of this matrix with some other matrices (see [4, Theorem 1]). The determinant of this matrix was computed by Kanemitsu and Kuzumaki (see [14]):

$$
\begin{aligned}
\operatorname{det} & \left(R_{p}^{\prime}\left(a b^{-1}\right)\right)_{a, b \in\{1, \ldots,(p-1) / 2\}} \\
= & \begin{cases}p^{(p-3) / 2} 2^{g_{2}\left(K_{p}\right)-1} h_{K_{p}}^{-} & \text {if } g_{2}\left(K_{p}\right)=g_{2}\left(K_{p}^{+}\right), \\
0 & \text { if } g_{2}\left(K_{p}\right)=2 g_{2}\left(K_{p}^{+}\right) .\end{cases}
\end{aligned}
$$

Another matrix connected with the relative class number of imaginary abelian fields is the Demjanenko matrix. For a positive integer $m$ and any integer $a$ let

$$
c_{m}(a)= \begin{cases}1 & \text { if }\left\langle\frac{a}{m}\right\rangle<\frac{1}{2} \\ 0 & \text { otherwise. }\end{cases}
$$

In [10] Hazama obtained for any odd prime $p$ the following formula

$$
\begin{aligned}
\operatorname{det}\left(c_{p}(a b)\right)_{a, b \in\{1, \ldots,(p-1) / 2\}} & =2 \cdot \operatorname{det}\left(c_{p}(a b)-\frac{1}{2}\right)_{a, b \in\{1, \ldots,(p-1) / 2\}} \\
& = \pm h_{K_{p}}^{-} \cdot p^{-1} \cdot F_{2, K_{p}}
\end{aligned}
$$

where $F_{2, K_{p}}$ is a constant depending on the decomposition of 2 in the $p$ th cyclotomic field (see Notation). The relationship between Demjanenko 
matrix and the Stickelberger ideal of the $p$-th cyclotomic field was described by Skula in [25] and [26].

Later on Girstmair in [7], Schwarz in [21], Sands and Schwarz in [20], and Dohmae in [3] generalized the formula (9) to an imaginary subfield of $p$-th cyclotomic field with a prime $p \equiv 3(\bmod 4)$, to an odd prime power cyclotomic field, to an imaginary field with an odd prime power conductor, and to an imaginary field with an odd conductor, respectively.

Finally in 1996 the notion of Demjanenko matrix was generalized to any imaginary abelian field $K$ by Hirabayashi (see [12]). We can rephrase his result as follows. Let $m$ be the conductor of $K$; for any automorphism $\sigma \in \operatorname{Gal}(K / \mathbb{Q})$ put

$$
d_{\sigma}=\sum_{\substack{j \bmod ^{\times} m \\(j, K)=\sigma}}\left(c_{m}(j)-\frac{1}{2}\right)
$$

where $j$ in the sum runs over the same set as in (5). Let $C$ be a system of representatives of $\operatorname{Gal}(K / \mathbb{Q}) / \operatorname{Gal}\left(K / K^{+}\right)$. Then Hirabayashi proved that

$$
\operatorname{det}\left(d_{\sigma \tau^{-1}}\right)_{\sigma, \tau \in C}=e_{K} \cdot F_{2, K} \cdot q_{K} \cdot \frac{h_{K}^{-}}{Q_{K} w_{K}},
$$

where $e_{K}, F_{2, K}, q_{K}$ can be easily described by means of odd Dirichlet characters corresponding to $K$ (see Notation).

Recently Tsumura and Hirabayashi independently discovered formulae generalizating both Maillet's and Demjanenko determinants (see [28] and [13]). Although their formulae look different, it can be shown (see Section 6 of this text) that in fact Tsumura's formula is a special case of Hirabayashi's one.

It is easy to show that Hirabayashi's formula can be read as follows. Let $K$ be an imaginary abelian field of conductor $m$, let $b$ be an integer which is not divisible by $m$. For any automorphism $\sigma \in \operatorname{Gal}(K / \mathbb{Q})$ put

$$
t_{\sigma}=\sum_{\substack{j \bmod ^{\times} m \\(j, K)=\sigma}}\left(\left[b\left\langle\frac{j}{m}\right\rangle\right]-\frac{b-1}{2}\right),
$$

where the sum is taken over the same set as in (5) and $[x]=x-\langle x\rangle$ means the integral part of a rational number $x$. Hirabayashi proved that

$$
\operatorname{det}\left(t_{\sigma \tau^{-1}}\right)_{\sigma, \tau \in C}=(-1)^{\left[K^{+}: \mathbb{Q}\right]} \cdot c_{K}(b) \cdot \frac{h_{K}^{-}}{Q_{K} w_{K}}
$$


where $c_{K}(b)$ can be described by means of odd Dirichlet characters corresponding to $K$ (see Notation). It is a straightforward calculation to show that taking $b=m+1$ we obtain (6) and putting $b=2$ we arrive at (11).

Let us mention that the determinant (13) for $m$ being an odd prime and $2 \leq b<m$ was already computed by Agoh and Skula in [1, Theorem 4.4 and Proposition 4.5] by means of the Stickelberger ideal.

There is another determinant connected with the relative class number of an imaginary abelian field which can be found in the literature. Let $p>q$ be odd primes and let $M$ be the set of all positive integers $a<p q$ relatively prime to $p q$. Then for $T=\left\{a \in M \mid\left\langle\frac{a}{p}\right\rangle\left\langle\left\langle\frac{a}{q}\right\rangle\right\}\right.$ Hazama in [11] proved that

$$
\begin{aligned}
\left|\operatorname{det}\left(H_{a, b}\right)_{a, b \in T}\right| & =2 \cdot\left|\operatorname{det}\left(H_{a, b}-\frac{1}{2}\right)_{a, b \in T}\right| \\
& =\frac{1}{2 p q} \cdot h_{K_{p q}}^{-} \cdot F_{p, K_{q}} \cdot F_{q, K_{p}}
\end{aligned}
$$

where

$$
H_{a, b}= \begin{cases}1 & \text { if }\left\langle\frac{a b}{p}\right\rangle<\left\langle\frac{a b}{q}\right\rangle \\ 0 & \text { otherwise. }\end{cases}
$$

In the rest of this paper we show a way of obtaining all the above mentioned formulae in a unique fashion (see Theorem 1 in Section 4). Moreover some new non-vanishing formulae are derived (e.g., see Example 4 in Section 4).

\section{§. Notation}

$\mathbb{Z}, \mathbb{Q}$, and $\mathbb{C}$ means the integers, the rational numbers, and the complex numbers, respectively.

$K_{m}$ denotes the $m$-th cyclotomic field (for any positive integer $m \not \equiv 2$ $(\bmod 4))$.

By an abelian field we have in mind a finite abelian extension of $\mathbb{Q}$. For any imaginary abelian field $K$ we define

$m$ the conductor of $K$;

$[K: \mathbb{Q}]$ the degree of $K$;

$h_{K}^{-}$the relative class number of $K$ (i.e., the quotient of the class number of $K$ by the class number of its maximal real subfield $K^{+}$);

$Q_{K}$ the Hasse unit index of $K$ (i.e., the index of the subgroup generated by all real units of $K$ and by all roots of unity in $K$ in the full group of units of $K)$; 
$w_{K}$ the number of roots of unity in $K$;

$X$ the group of all Dirichlet characters corresponding to $K$, (all characters are supposed to be primitive);

$X^{-}=\{\chi \in X ; \chi(-1)=-1\}$ the set of all odd Dirichlet characters corresponding to $K$;

for any rational prime $p$ we introduce:

$e_{p}(K)$ the ramification index of $p$ in $K$;

$f_{p}(K)$ the residue class degree of $p$ in $K$;

$g_{p}(K)$ the number of primes in $K$ dividing $p$;

$\psi_{p}(K)= \begin{cases}0 & \text { if } e_{p}(K)=2 e_{p}\left(K^{+}\right), \\ 1 & \text { if } g_{p}(K)=2 g_{p}\left(K^{+}\right), \\ -1 & \text { if } f_{p}(K)=2 f_{p}\left(K^{+}\right) ;\end{cases}$

$F_{p, K}=\prod_{\chi \in X^{-}}(p-\chi(p))=p^{\left[K^{+}: \mathbb{Q}\right]}\left(1-\psi_{p}(K) p^{-f_{p}\left(K^{+}\right)}\right)^{g_{p}\left(K^{+}\right)}$, the second equality can be obtained by a standard technique (see [17, Lemma 6] or [30, Theorem 3.7]);

$q_{K}=\prod_{\chi \in X^{-}} \prod_{p \mid m}(1-\chi(p))$, where the second product is taken over all rational primes dividing $m$; by the mentioned technique one can show that $q_{K}=$ 0 if there is a prime $p \mid m$ satisfying $g_{p}(K)=2 g_{p}\left(K^{+}\right)$and $q_{K}=2^{n}$ otherwise, where $n$ is the sum of all $g_{p}(K)$ such that $p \mid m$ and $e_{p}(K)=$ $e_{p}\left(K^{+}\right)$;

$e_{K}= \begin{cases}0 & \text { if } m \text { is even and there is } \chi \in X^{-} \text {such that } \chi(1+(m / 2))=1, \\ 1 & \text { otherwise. }\end{cases}$

For an integer $b$ which is not divisible by $m$ put $m^{\prime}=m /(m, b), b^{\prime}=$ $b /(m, b)$, and

$c_{K}(b)=\prod_{\chi \in X^{-}} c_{K}^{\chi}(b)$. Here for any character $\chi \in X^{-}$of conductor $f_{\chi}$ we define

$c_{K}^{\chi}(b)= \begin{cases}b \prod_{p \mid m}(1-\bar{\chi}(p)) & \text { if } f_{\chi} \nmid m^{\prime}, \\ b \prod_{p \mid m}(1-\bar{\chi}(p))-\frac{\varphi(m)}{\varphi\left(m^{\prime}\right)} \chi\left(b^{\prime}\right) \prod_{p \mid m^{\prime}}(1-\bar{\chi}(p)) & \text { if } f_{\chi} \mid m^{\prime},\end{cases}$

where $\varphi$ means the Euler $\varphi$-function. 


\section{$\S 3$. Stickelberger ideal}

In this section we shall define the Stickelberger ideal of an abelian field following Sinnott (see [23]) and derive some basic facts which will prove useful in the next section.

For any abelian field $L$ let $G_{L}=\operatorname{Gal}(L / \mathbb{Q})$. If $M / L$ is an extension of abelian fields, then $\operatorname{res}_{M / L}: \mathbb{C}\left[G_{M}\right] \rightarrow \mathbb{C}\left[G_{L}\right]$ is the ring homomorphism of group rings induced by restriction; $\operatorname{cor}_{M / L}: \mathbb{C}\left[G_{L}\right] \rightarrow \mathbb{C}\left[G_{M}\right]$ is the additive homomorphism which maps any $\sigma \in G_{L}$ to the sum of all automorphisms of $M$ whose restriction to $L$ is $\sigma$.

For any abelian field $M$ and any character $\chi$ of $G_{M}$ we also denote by $\chi$ the associated Dirichlet character and by $\rho_{M}^{\chi}$ the ring homomorphism $\rho_{M}^{\chi}$ : $\mathbb{C}\left[G_{M}\right] \rightarrow \mathbb{C}$ induced by $\chi$. We say that a Dirichlet character corresponds to $M$ if it is associated to a character of $G_{M}$. Recall that all Dirichlet characters are supposed to be primitive.

LEMma 1. Let $M$ and $L$ be abelian fields, $L \subseteq M$; let $\chi$ be a Dirichlet character corresponding to $M$, and let $\alpha \in \mathbb{C}\left[G_{L}\right], \beta \in \mathbb{C}\left[G_{M}\right]$.

1. If $\chi$ does not correspond to $L$, then $\rho_{M}^{\chi}\left(\operatorname{cor}_{M / L} \alpha\right)=0$.

2. If $\chi$ corresponds to $L$, then

$$
\begin{aligned}
\rho_{M}^{\chi}\left(\operatorname{cor}_{M / L} \alpha\right) & =[M: L] \rho_{L}^{\chi}(\alpha), \\
\rho_{L}^{\chi}\left(\operatorname{res}_{M / L} \beta\right) & =\rho_{M}^{\chi}(\beta),
\end{aligned}
$$

where $[M: L]$ means the relative degree of $M$ over $L$.

Proof. Follows directly from the definitions.

LEMma 2. Let $K$ be an imaginary abelian field, let $j \in G_{K}$ be the complex conjugation, and let $C$ be a system of representatives of $G_{K} /\{1, j\}$. Let us fix $\theta \in(1-j) \mathbb{Q}\left[G_{K}\right]$ and define $t_{\sigma}$ by $\theta=\sum_{\sigma \in G_{K}} t_{\sigma} \sigma^{-1}$. Then we have

$$
\operatorname{det}\left(t_{\sigma \tau^{-1}}\right)_{\sigma, \tau \in C}=\prod_{\chi \in X^{-}} \frac{1}{2} \rho_{K}^{\chi}(\theta)
$$

where $X^{-}$is the set of all odd Dirichlet characters corresponding to $K$.

Proof. For any $\tau \in G_{K}$ we have

$$
\theta \frac{1-j}{2} \tau^{-1}=\theta \tau^{-1}=\sum_{\sigma \in G_{K}} t_{\sigma \tau^{-1}} \sigma^{-1}=\sum_{\sigma \in C} 2 t_{\sigma \tau^{-1}} \frac{1-j}{2} \sigma^{-1}
$$


so $\left(2 t_{\sigma \tau^{-1}}\right)_{\sigma, \tau \in C}$ is the transition matrix from $\left\{\theta \frac{1-j}{2} \tau^{-1} ; \tau \in C\right\}$ to $\left\{\frac{1-j}{2} \tau^{-1} ; \tau \in C\right\}$. The lemma follows from Lemma 1.2(b) in [23]. (Alternatively, the orthogonal idempotents $\frac{1}{\# G} \sum_{\sigma \in G} \chi(\sigma) \sigma^{-1} \in \mathbb{C}\left[G_{K}\right]$ corresponding to odd characters $\chi \in X^{-}$form a basis of $(1-j) \mathbb{C}\left[G_{K}\right]$. The matrix of the linear transformation given by the multiplication by $\theta$ with respect to this basis is the diagonal matrix $\operatorname{diag}\left(\rho_{K}^{\chi}(\theta)\right)_{\chi \in X^{-}}$.)

For any positive integer $n$ let $K_{n}$ be the $n$-th cyclotomic field. Following Sinnott, let us define for any integer $a$ the element

$$
\theta_{n}(a)=\sum_{t \bmod ^{\times} n}\left\langle-\frac{a t}{n}\right\rangle\left(t, K_{n}\right)^{-1} \in \mathbb{Q}\left[G_{K_{n}}\right]
$$

here the sum is taken over a reduced residue system modulo $n,\langle x\rangle$ is the fractional part of a real number $x$ and $\left(t, K_{n}\right) \in G_{K_{n}}$ is the automorphism sending any root of unity to its $t$-th power.

Let $K$ be an abelian field. For any positive integer $n$ and any integer $a$ let

$$
\theta_{n}^{\prime}(a)=\operatorname{cor}_{K / K \cap K_{n}} \operatorname{res}_{K_{n} / K \cap K_{n}} \theta_{n}(a) \in \mathbb{Q}\left[G_{K}\right] .
$$

The Stickelberger ideal of $K$ is the intersection $S=S^{\prime} \cap \mathbb{Z}\left[G_{K}\right]$, where $S^{\prime}$ is the additive subgroup of $\mathbb{Q}\left[G_{K}\right]$ generated by

$$
\left\{\theta_{n}^{\prime}(a) ; a, n \in \mathbb{Z}, n \geq 1\right\} .
$$

Lemma 3. Let $n$ be a positive integer, let a be an integer relatively prime to $n$ and let $\chi$ be a non-trivial Dirichlet character whose conductor divides $n$. Then

$$
\rho_{K_{n}}^{\chi}\left(\theta_{n}(a)\right)=\chi(-a) B_{1, \bar{\chi}} \prod_{p \mid n}(1-\bar{\chi}(p))
$$

where $B_{1, \chi}$ means the first generalized Bernoulli number.

Proof. We have

$$
\rho_{K_{n}}^{\chi}\left(\theta_{n}(a)\right)=\rho_{K_{n}}^{\chi}\left(\left(-a, K_{n}\right) \sum_{t \bmod ^{\times} n}\left\langle\frac{t}{n}\right\rangle\left(t, K_{n}\right)^{-1}\right)=\frac{\chi(-a)}{n} \sum_{\substack{t=1 \\(t, n)=1}}^{n} t \bar{\chi}(t) .
$$


Let $f_{\chi}$ be the conductor of $\chi$. The well-known distribution relations give

$$
\frac{1}{n} \sum_{\substack{t=1 \\(t, n)=1}}^{n} t \bar{\chi}(t)=\left(\frac{1}{f_{\chi}} \sum_{t=1}^{f_{\chi}} t \bar{\chi}(t)\right) \prod_{p \mid n}(1-\bar{\chi}(p))=B_{1, \bar{\chi}} \prod_{p \mid n}(1-\bar{\chi}(p)),
$$

(e.g., see [9] or [22]) and the lemma follows.

Lemma 4. Let $K$ be an imaginary abelian field, let $\chi$ be an odd Dirichlet character corresponding to $K, f_{\chi}$ being its conductor. For any positive integer $n$ and any integer a we have

$$
\rho_{K}^{\chi}\left(\theta_{n}^{\prime}(a)\right)= \begin{cases}0 & \text { if } f_{\chi} \nmid n, \\ {\left[K: K \cap K_{n}\right] \rho_{K_{n}}^{\chi}\left(\theta_{n}(a)\right)} & \text { if } f_{\chi} \mid n .\end{cases}
$$

Proof. Follows from the definition of $\theta_{n}^{\prime}(a)$ and Lemma 1.

\section{$\S 4$. Determinants obtained by means of the Stickelberger ideal}

For any imaginary abelian field $K$ and any integer $t$ relatively prime to the conductor $m$ of $K$, let $(t, K)$ be the automorphism given by the Artin map, i.e., $(t, K)$ is the restriction to $K$ of the automorphism of the $m$-th cyclotomic field $K_{m}$ which sends each root of unity to its $t$-th power.

THEOREM 1. Let $K$ be an imaginary abelian field of conductor $m$ and let $G=\operatorname{Gal}(K / \mathbb{Q})$ be its Galois group. Let $a_{n, \sigma}$ be rational numbers for any divisor $n>1$ of $m$ and any $\sigma \in G$. We put

$$
t_{\sigma}=\sum_{\substack{n \mid m \\ n>1}} \sum_{t \bmod ^{\times} m}\left(\frac{1}{2}-\left\langle\frac{t}{n}\right\rangle\right) a_{n,(t, K) \sigma^{-1}}
$$

for any $\sigma \in G$, where the second sum is taken over a reduced residue system modulo $m$. Let $j \in G$ be the complex conjugation. Then for any system $C$ of representatives of $G /\{1, j\}$, we have

$$
\operatorname{det}\left(t_{\sigma \tau^{-1}}\right)_{\sigma, \tau \in C}=\frac{h_{K}^{-}}{Q_{K} w_{K}} \prod_{\chi \in X^{-}} \sum_{\substack{n\left|m \\ f_{\chi}\right| n}} b_{n, \chi} \prod_{p \mid n}(1-\bar{\chi}(p))
$$

where $h_{K}^{-}, Q_{K}$, and $w_{K}$ are the relative class number of $K$, the Hasse unit index of $K$, and the number of roots of unity in $K$, respectively, $X^{-}$is the 
set of all odd primitive Dirichlet characters corresponding to $K, f_{\chi}$ is the conductor of $\chi$, and

$$
b_{n, \chi}=\frac{\varphi(m)}{\varphi(n)} \sum_{\sigma \in G} a_{n, \sigma} \chi(\sigma) .
$$

Proof. For any divisor $n>1$ of $m$, let us denote

$$
d_{n}=\left[K_{m}: K K_{n}\right] \sum_{\sigma \in G} a_{n, \sigma} \sigma \in \mathbb{Q}[G],
$$

where $K K_{n}$ means the compositum of these two fields. We put

$$
\theta=\frac{1-j}{2} \sum_{1<n \mid m} d_{n} \theta_{n}^{\prime}(1)
$$

Then we have (e.g., see [16, Lemma 14])

$$
\begin{aligned}
{\left[K_{m}: K K_{n}\right] \theta_{n}^{\prime}(1) } & =\left[K_{m}: K K_{n}\right] \operatorname{cor}_{K / K \cap K_{n}} \operatorname{res}_{K_{n} / K \cap K_{n}} \theta_{n}(1) \\
& =\operatorname{res}_{K_{m} / K} \operatorname{cor}_{K_{m} / K_{n}} \theta_{n}(1) \\
& =\sum_{t \bmod _{m} m}\left\langle-\frac{t}{n}\right\rangle(t, K)^{-1} ;
\end{aligned}
$$

therefore

$$
\begin{aligned}
\theta & =\frac{1-j}{2} \sum_{1<n \mid m}\left[K_{m}: K K_{n}\right] \theta_{n}^{\prime}(1) \sum_{\sigma \in G} a_{n, \sigma} \sigma \\
& =\frac{1-j}{2} \sum_{1<n \mid m} \sum_{\sigma \in G} a_{n, \sigma} \sigma \sum_{t \bmod ^{\times} m}\left\langle-\frac{t}{n}\right\rangle(t, K)^{-1} \\
& =\frac{1}{2} \sum_{1<n \mid m} \sum_{t \bmod ^{\times}} \sum_{\sigma \in G}\left(\left\langle-\frac{t}{n}\right\rangle-\left\langle\frac{t}{n}\right\rangle\right) a_{n, \sigma} \sigma(t, K)^{-1} \\
& =\sum_{\sigma \in G} t_{\sigma} \sigma^{-1},
\end{aligned}
$$

since $\left\langle-\frac{t}{n}\right\rangle+\left\langle\frac{t}{n}\right\rangle=1$. Lemma 2 gives

$$
\operatorname{det}\left(t_{\sigma \tau^{-1}}\right)_{\sigma, \tau \in C}=\prod_{\chi \in X^{-}} \frac{1}{2} \rho_{K}^{\chi}(\theta) .
$$


Now

$$
\rho_{K}^{\chi}(\theta)=\sum_{1<n \mid m} \rho_{K}^{\chi}\left(d_{n}\right) \rho_{K}^{\chi}\left(\theta_{n}^{\prime}(1)\right)=\sum_{\substack{n\left|m \\ f_{\chi}\right| n}} \rho_{K}^{\chi}\left(d_{n}\right)\left[K: K \cap K_{n}\right] \rho_{K_{n}}^{\chi}\left(\theta_{n}(1)\right)
$$

due to Lemma 4. Since $\left[K: K \cap K_{n}\right]=\left[K K_{n}: K_{n}\right]$, we have $\rho_{K}^{\chi}\left(d_{n}\right)[K$ : $\left.K \cap K_{n}\right]=b_{n, \chi}$, and Lemma 3 implies

$$
\rho_{K}^{\chi}(\theta)=-B_{1, \bar{\chi}} \sum_{\substack{n\left|m \\ f_{\chi}\right| n}} b_{n, \chi} \prod_{p \mid n}(1-\bar{\chi}(p))
$$

Therefore

$$
\operatorname{det}\left(t_{\sigma \tau^{-1}}\right)_{\sigma, \tau \in C}=\prod_{\chi \in X^{-}}\left(-\frac{1}{2} B_{1, \bar{\chi}}\right) \sum_{\substack{n\left|m \\ f_{\chi}\right| n}} b_{n, \chi} \prod_{p \mid n}(1-\bar{\chi}(p))
$$

The theorem follows using the analytic class number formula

$$
h_{K}^{-}=Q_{K} w_{K} \prod_{\chi \in X^{-}}\left(-\frac{1}{2} B_{1, \chi}\right)
$$

(e.g., see [30, Theorem 4.17]).

Now we can choose any rational numbers $a_{n, \sigma}$ and obtain a determinant formula for the relative class number. As examples we will show how to obtain the formulae mentioned in the introduction. We will keep the assumption that $K$ is an imaginary abelian field of conductor $m$.

EXAMPLE 1. Let $b$ be an integer which is not divisible by $m$. We put $m^{\prime}=m /(m, b), b^{\prime}=b /(m, b)$ and we choose and fix some $b^{\prime \prime}$ which is relatively prime to $m$ and satisfies $b^{\prime \prime} \equiv b^{\prime}\left(\bmod m^{\prime}\right)$. For any divisor $n>1$ of $m$ and any $\sigma \in G$ we define $a_{n, \sigma}=a_{n, \sigma}^{\prime}+a_{n, \sigma}^{\prime \prime}$, where

$$
\begin{aligned}
& a_{n, \sigma}^{\prime}= \begin{cases}-b & \text { if } n=m \text { and } \sigma=(1, K), \\
0 & \text { otherwise, }\end{cases} \\
& a_{n, \sigma}^{\prime \prime}= \begin{cases}1 & \text { if } n=m^{\prime} \text { and } \sigma=\left(b^{\prime \prime}, K\right), \\
0 & \text { otherwise. }\end{cases}
\end{aligned}
$$


The definition in Theorem 1 gives

$$
\begin{aligned}
t_{\sigma} & =\sum_{\substack{t \bmod ^{\times} m \\
(t, K)=\sigma}}(-b)\left(\frac{1}{2}-\left\langle\frac{t}{m}\right\rangle\right)+\sum_{\substack{t \bmod ^{\times} m \\
(t, K)\left(b^{\prime \prime}, K\right)^{-1}=\sigma}}\left(\frac{1}{2}-\left\langle\frac{t}{m^{\prime}}\right\rangle\right) \\
& =\sum_{\substack{t \bmod ^{\times} m \\
(t, K)=\sigma}}(-b)\left(\frac{1}{2}-\left\langle\frac{t}{m}\right\rangle\right)+\sum_{\substack{t \bmod ^{\times} m \\
(t, K)=\sigma}}\left(\frac{1}{2}-\left\langle\frac{t b^{\prime \prime}}{m^{\prime}}\right\rangle\right) .
\end{aligned}
$$

$\operatorname{But}\left\langle\frac{t b^{\prime \prime}}{m^{\prime}}\right\rangle=\left\langle\frac{t b^{\prime}}{m^{\prime}}\right\rangle=\left\langle\frac{t b}{m}\right\rangle$, so

$$
\begin{aligned}
t_{\sigma} & =\sum_{\substack{t \bmod ^{\times} m \\
(t, K)=\sigma}}\left((-b)\left(\frac{1}{2}-\left\langle\frac{t}{m}\right\rangle\right)+\left(\frac{1}{2}-\left\langle\frac{t b}{m}\right\rangle\right)\right) \\
& =\sum_{\substack{t \bmod ^{\times} m \\
(t, K)=\sigma}}\left(\left[b\left\langle\frac{t}{m}\right\rangle\right]-\frac{b-1}{2}\right),
\end{aligned}
$$

which is (12). An easy straightforward calculation gives that

$$
\sum_{\substack{n\left|m \\ f_{\chi}\right| n}} b_{n, \chi} \prod_{p \mid n}(1-\bar{\chi}(p))=-c_{K}^{\chi}(b)
$$

(see Notation for the definition of $\left.c_{K}^{\chi}(b)\right)$ and Theorem 1 gives (13).

EXAMPLE 2. Let us generalize formula (7) now. For an integer a let $R_{m}^{\prime}(a)$ mean the integer determined by $R_{m}^{\prime}(a) \equiv a(\bmod m)$ and $-m / 2 \leq$ $R_{m}^{\prime}(a)<m / 2$. It is easy to see that $\frac{1}{m} R_{m}^{\prime}(a)=\left\langle\frac{a}{m}+\frac{1}{2}\right\rangle-\frac{1}{2}$. Hence we shall compute the determinant ${ }^{2}$

$$
\operatorname{det}\left(t_{\sigma \tau^{-1}}\right)_{\sigma, \tau \in C}
$$

for a system $C$ of representatives of $G /\{1, j\}$ where

$$
t_{\sigma}=\sum_{\substack{t \bmod ^{\times} m \\(t, K)=\sigma}}\left(\left\langle\frac{t}{m}+\frac{1}{2}\right\rangle-\frac{1}{2}\right)
$$

\footnotetext{
${ }^{2}$ Let us notice that this determinant was computed by Kanemitsu and Kuzumaki but their formula seems to be valid only for cyclotomic fields of an odd conductor (see [14, Theorem 1, formula for $\left.\left.D_{S, 1}^{\prime}(0)\right]\right)$.
} 
for any $\sigma \in G$. We shall distinguish two cases depending on the parity of $m$. At first, let us suppose that $m$ is even. Then $m$ is divisible by 4 because $m$ is the conductor of $K$; hence $1+\frac{m}{2}$ is relatively prime to $m$. For an odd integer $t$ we have $\left\langle\frac{t}{m}+\frac{1}{2}\right\rangle=\left\langle\frac{t}{m}+\frac{t}{2}\right\rangle=\left\langle\frac{t}{m}\left(1+\frac{m}{2}\right)\right\rangle$ and so

$$
t_{\sigma}=\sum_{\substack{t \bmod ^{\times} m \\(t, K)\left(1+\frac{m}{2}, K\right)^{-1}=\sigma}}\left(\left\langle\frac{t}{m}\right\rangle-\frac{1}{2}\right) .
$$

Therefore (16) will be satisfied if we put

$$
a_{n, \sigma}= \begin{cases}-1 & \text { if } n=m \text { and } \sigma=\left(1+\frac{m}{2}, K\right) \\ 0 & \text { otherwise. }\end{cases}
$$

Then for any $\chi \in X^{-}$we have $b_{m, \chi}=-\chi\left(1+\frac{m}{2}\right)$ and $b_{n, \chi}=0$ if $n \neq m$. Theorem 1 gives

$$
\operatorname{det}\left(t_{\sigma \tau^{-1}}\right)_{\sigma, \tau \in C}=\frac{h_{K}^{-}}{Q_{K} w_{K}} \prod_{\chi \in X^{-}}\left(-\chi\left(1+\frac{m}{2}\right) \prod_{p \mid m}(1-\chi(p))\right) .
$$

There is $\chi \in X$ such that $\chi\left(1+\frac{m}{2}\right) \neq 1$ because $m$ is the conductor of $K$. Moreover $\chi\left(1+\frac{m}{2}\right)^{2}=\chi\left(1+m+\frac{m^{2}}{4}\right)=1$ for any $\chi \in X$ so we get that $Y=\left\{\chi \in X ; \chi\left(1+\frac{m}{2}\right)=1\right\}$ is a subgroup of $X$ of index 2 . Now $X^{+}=Y$ if and only if the conductor $m^{+}$of $K^{+}$divides $m / 2$. Hence

$$
\operatorname{det}\left(t_{\sigma \tau^{-1}}\right)_{\sigma, \tau \in C}=\frac{h_{K}^{-}}{Q_{K} w_{K}} q_{K} z_{K}
$$

where $z_{K}=1$ if $m^{+} \mid(m / 2)$ and $z_{K}=(-1)^{[K: \mathbb{Q}] / 4}$ if $m^{+} \nmid(m / 2)$; (see Notation for the definition of $q_{K}$ ).

Now we begin to consider the case $m$ being odd. Because $\langle 2 x\rangle=\langle x\rangle+$ $\left\langle x+\frac{1}{2}\right\rangle-\frac{1}{2}$ for any real $x$, we have

$$
t_{\sigma}=\sum_{\substack{t \bmod ^{\times} m \\(t, K)=\sigma}}\left(\left\langle\frac{2 t}{m}\right\rangle-\left\langle\frac{t}{m}\right\rangle\right)
$$

for any $\sigma \in G$. Thus (16) will be satisfied if

$$
a_{n, \sigma}= \begin{cases}1 & \text { if } n=m \text { and } \sigma=(1, K) \neq(2, K) \\ -1 & \text { if } n=m \text { and } \sigma=(2, K) \neq(1, K) \\ 0 & \text { otherwise }\end{cases}
$$


Then $b_{m, \chi}=1-\chi(2)$ and $b_{n, \chi}=0$ if $n \neq m$ and Theorem 1 gives

$$
\begin{aligned}
\operatorname{det}\left(t_{\sigma \tau^{-1}}\right)_{\sigma, \tau \in C} & =\frac{h_{K}^{-}}{Q_{K} w_{K}} \prod_{\chi \in X^{-}}\left((1-\chi(2)) \prod_{p \mid m}(1-\chi(p))\right) \\
& =\frac{h_{K}^{-}}{Q_{K} w_{K}} q_{K} z_{K},
\end{aligned}
$$

where $z_{K}=0$ if $\psi_{2}(K)=1$ and $z_{K}=2^{g_{2}\left(K^{+}\right)}$otherwise (see Notation).

Remark. Let us mention the obvious fact that the determinant (17) is not zero if and only if the $\mathbb{Q}[G]$-module generated by $\theta$ equals $(1-j) \mathbb{Q}[G]$. Having $\theta \in S$ this condition means that all conjugates of $\theta$ generate a subideal of finite index in the "minus part" of the Stickelberger ideal $S^{-}=$ $S \cap(1-j) \mathbb{Q}[G]$. Therefore we can obtain a non-vanishing determinant formula for the relative class number just taking such an element of the Stickelberger ideal (or a rational multiple of this element). An element of this kind has been used by Lettl to explain the analogy between Girstmair's cotangent numbers and the Stickelberger ideal (see [6] and [17]). The nonvanishing class number formula given by this element is computed in the following example.

EXAMPLE 3. Let us consider the prime decomposition $m=\prod_{i=1}^{s} p_{i}^{r_{i}}$, with pairwise different primes $p_{1}, \ldots, p_{s}$ and positive integers $r_{1}, \ldots, r_{s}$. For any $I \subseteq\{1, \ldots, s\}$ let $m_{I}=\prod_{i \in I} p_{i}^{r_{i}}$. For any $i \in\{1, \ldots, s\}$ we fix the Frobenius automorphism by Frob $p_{i}=\left(p_{i}^{\prime}, K\right)^{-1}$, where $p_{i}^{\prime} \equiv 1\left(\bmod p_{i}^{r_{i}}\right)$ and $p_{i}^{\prime} p_{i} \equiv 1\left(\bmod m_{\{1, \ldots, s\} \backslash\{i\}}\right)$. For any divisor $n>1$ of $m$ and any $\sigma \in G$ we define $(i \notin I$ means $i \in\{1, \ldots, s\} \backslash I)$

$$
a_{n, \sigma}= \begin{cases}\prod_{i \notin I}\left(-p_{i}^{1-r_{i}}\right) & \text { if there is } I \subseteq\{1, \ldots, s\} \text { such that } n=m_{I} \\ 0 & \text { and } \sigma=\prod_{i \notin I}\left(\text { Frob } p_{i}\right)^{-1}, \\ \text { otherwise. }\end{cases}
$$

Then (16) gives (an empty product equals 1)

$$
\begin{aligned}
t_{\sigma} & =\sum_{\emptyset \neq I \subseteq\{1, \ldots, s\}} \sum_{\substack{t \bmod ^{\times} m \\
(t, K) \sigma^{-1}=\prod_{i \notin I}\left(p_{i}^{\prime}, K\right)}}\left(\frac{1}{2}-\left\langle\frac{t}{m_{I}}\right\rangle\right) \prod_{i \notin I}\left(-p_{i}^{1-r_{i}}\right) \\
& =\sum_{\emptyset \neq I \subseteq\{1, \ldots, s\}} \sum_{\substack{t \bmod ^{\times} m \\
\sigma=(t, K)}}\left(\frac{1}{2}-\left\langle\frac{t \prod_{i \notin I} p_{i}^{\prime}}{m_{I}}\right\rangle\right) \prod_{i \notin I}\left(-p_{i}^{1-r_{i}}\right) .
\end{aligned}
$$


Theorem 1 states

$$
\begin{aligned}
& \operatorname{det}\left(t_{\sigma \tau^{-1}}\right)_{\sigma, \tau \in C} \\
& =\frac{h_{K}^{-}}{Q_{K} w_{K}} \prod_{\substack{\chi \in X^{-} \\
I \subseteq\{1, \ldots, s\} \\
f_{\chi} \mid m_{I}}} \prod_{i \notin I}\left(\varphi\left(p_{i}^{r_{i}}\right) \bar{\chi}\left(p_{i}\right)\left(-p_{i}^{1-r_{i}}\right)\right) \prod_{i \in I}\left(1-\bar{\chi}\left(p_{i}\right)\right) \\
& =\frac{h_{K}^{-}}{Q_{K} w_{K}} \prod_{\chi \in X^{-}} \prod_{\substack{i \in\{1, \ldots, s\} \\
p_{i} \nmid f_{\chi}}}\left(1-p_{i} \bar{\chi}\left(p_{i}\right)\right) \\
& =\frac{h_{K}^{-}}{Q_{K} w_{K}} \prod_{p \mid m}\left(1-\psi_{p}(K) p^{f_{p}\left(K^{+}\right)}\right)^{g_{p}\left(K^{+}\right)}
\end{aligned}
$$

(see Notation). Let us suppose that $m$ is square-free (i.e., $K$ is not wildly ramified). Then the formula for $t_{\sigma}$ can be simplified as follows

$$
t_{\sigma}=\sum_{\emptyset \neq I \subseteq\{1, \ldots, s\}} \sum_{\substack{\left.t \bmod _{\sigma=(t, K)}^{\times} \\ t-1\right)^{s-|I|}}}\left(\frac{1}{2}-\left\langle\frac{t \prod_{i \notin I} p_{i}^{\prime} p_{i}}{m}\right\rangle\right) .
$$

It is easy to see that if $s>1$ then $t_{\sigma}+\frac{1}{2}$ is an integer and $\left|t_{\sigma}\right|<\frac{1}{2}\left(2^{s}-1\right)\left[K_{m}\right.$ : $K]$.

Finally, let us consider the case of cyclotomic field $K_{m}$, where $m=p q$ and $p>q$ are primes. Then we have $t_{\sigma}= \pm 1 / 2$. Let us fix an integer $d$ such that $d \equiv p(\bmod q)$ and $d \equiv-q(\bmod p)$. For any integer $a$ relatively prime to $m$ we have

$$
t_{(a d, K)}=\left(\frac{1}{2}-\left\langle\frac{a d}{m}\right\rangle\right)-\left(\frac{1}{2}-\left\langle\frac{a}{q}\right\rangle\right)-\left(\frac{1}{2}-\left\langle-\frac{a}{p}\right\rangle\right) .
$$

Since $t_{(a d, K)}= \pm 1 / 2$ and $\left|\frac{1}{2}-\left\langle\frac{a d}{m}\right\rangle\right|<1 / 2$, we have

$$
\begin{aligned}
t_{(a d, K)}=\frac{1}{2} & \Longleftrightarrow\left(\frac{1}{2}-\left\langle\frac{a}{q}\right\rangle\right)+\left(\frac{1}{2}-\left\langle-\frac{a}{p}\right\rangle\right)<0 \\
& \Longleftrightarrow\left\langle\frac{a}{p}\right\rangle<\left\langle\frac{a}{q}\right\rangle \Longleftrightarrow H_{a, 1}=1
\end{aligned}
$$

(see $(15))$. Hence $t_{(a d, K)}=H_{a, 1}-\frac{1}{2}$ and we obtain Hazama's formula (14) by means of the permutation of the rows of the matrix. 
ExAmPLE 4. Another non-vanishing formula can be obtained by a modification of Ramachandra's construction of independent cyclotomic units (see [30, pp. 147 and 152]). As in the previous example let us decompose $m=\prod_{i=1}^{s} p_{i}^{r_{i}}$ with pairwise different primes $p_{1}, \ldots, p_{s}$ and positive integers $r_{1}, \ldots, r_{s}$. For any $I \subseteq\{1, \ldots, s\}$ let $m_{I}=\prod_{i \in I} p_{i}^{r_{i}}$. For any $i \in\{1, \ldots, s\}$ let us fix a rational number $c_{i}$ and put

$a_{n, \sigma}= \begin{cases}\prod_{i \notin I} c_{i} & \text { if there is } I \subseteq\{1, \ldots, s\} \text { such that } n=m_{I} \text { and } \sigma=1, \\ 0 & \text { otherwise. }\end{cases}$

Then (16) gives

$$
t_{\sigma}=\sum_{\emptyset \neq I \subseteq\{1, \ldots, s\}} \sum_{\substack{t \bmod ^{\times} m \\(t, K)=\sigma}}\left(\frac{1}{2}-\left\langle\frac{t}{m_{I}}\right\rangle\right) \prod_{i \notin I} c_{i}
$$

and Theorem 1 states

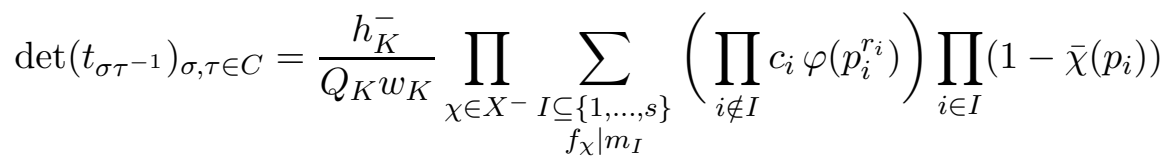

$$
\begin{aligned}
& =\frac{h_{K}^{-}}{Q_{K} w_{K}} \prod_{\chi \in X^{-}} \prod_{\substack{i \in\{1, \ldots, s\} \\
p_{i} \nmid f_{\chi}}}\left(c_{i} \varphi\left(p_{i}^{r_{i}}\right)+1-\bar{\chi}\left(p_{i}\right)\right) \\
& =\frac{h_{K}^{-}}{Q_{K} w_{K}} \prod_{\substack{i=1, \ldots, s \\
\psi_{p_{i}}(K) \neq 0}}\left(\left(c_{i} \varphi\left(p_{i}^{r_{i}}\right)+1\right)^{f_{p_{i}}\left(K^{+}\right)}-\psi_{p_{i}}(K)\right)^{g_{p_{i}}\left(K^{+}\right)} .
\end{aligned}
$$

For example, if we take $c_{1}=\cdots=c_{s}=0$ then we obtain (6). If we put $c_{i}=-\varphi\left(p_{i}^{r_{i}}\right)^{-1}$ then we get

$$
\begin{aligned}
& \operatorname{det}\left((-1)^{s} \sum_{\emptyset \neq I \subseteq\{1, \ldots, s\}}(-1)^{|I|} \frac{\varphi\left(m_{I}\right)}{\varphi(m)} \sum_{\begin{array}{c}
t \bmod ^{\times} m \\
(t, K)=\sigma \tau^{-1}
\end{array}}\left(\frac{1}{2}-\left\langle\frac{t}{m_{I}}\right\rangle\right)\right)_{\sigma, \tau \in C} \\
& =(-1)^{n} \frac{h_{K}^{-}}{Q_{K} w_{K}}
\end{aligned}
$$

where $n$ is the number of primes of $K^{+}$dividing $m$ which splits in $K / K^{+}$. 


\section{$\S 5$. Adding a constant}

We have covered all formulae mentioned in the introduction with the exception of (2) and (3). These determinants can be obtained by adding a constant to all entries of the determinant computed by Theorem 1.

In this section we keep the notation of the previous section and we still assume that $K$ is an imaginary abelian field, $G=\operatorname{Gal}(K / \mathbb{Q}), j \in G$ is the complex conjugation, $C$ a fixed system of representatives of $G /\{1, j\}$. Moreover we have chosen rational numbers $a_{n, \sigma}$ which give $t_{\sigma}$ and $b_{n, \chi}$ by means of (16) and (18), respectively, and $\theta=\sum_{\sigma \in G} t_{\sigma} \sigma^{-1}$. We define the polynomial

$$
D(x)=\operatorname{det}\left(x+t_{\sigma \tau^{-1}}\right)_{\sigma, \tau \in C} .
$$

This section is devoted to the computation of $D(x)$. Of course we know $D(0)$ which was determined by Theorem 1 .

First we need an easy lemma.

Lemma 5. Let $\delta_{i j}$ means the Kronecker delta. We have

$$
\operatorname{det}\left(x+\delta_{i j} a_{i}\right)_{i, j \in\{1, \ldots, n\}}=\left(\prod_{i=1}^{n} a_{i}\right)+x \sum_{i=1}^{n} \prod_{\substack{j=1, \ldots, n \\ j \neq i}} a_{j}
$$

for any complex numbers $a_{1}, \ldots, a_{n}$.

Proof. Is given by induction with respect to $n$. Let us denote $r_{n}\left(a_{1}, a_{2}, \ldots, a_{n}\right)=\operatorname{det}\left(x+\delta_{i j} a_{i}\right)_{i, j \in\{1, \ldots, n\}}$. If $n>1$ then after subtracting the first row from the last one, Laplace development with respect to the last row gives

$$
r_{n}\left(a_{1}, a_{2}, \ldots, a_{n}\right)=a_{1} r_{n-1}\left(0, a_{2}, \ldots, a_{n-1}\right)+a_{n} r_{n-1}\left(a_{1}, a_{2}, \ldots, a_{n-1}\right)
$$

and the lemma follows.

Proposition 1. We have

$$
D(x)-D(0)=-\frac{2 x}{d} \cdot \frac{h_{K}^{-}}{Q_{K} w_{K}} \sum_{\chi \in X^{-}} \frac{\left|c_{\chi}\right|^{2}}{B_{1, \chi}} \prod_{\substack{\psi \in X^{-} \\ \psi \neq \chi}} \sum_{\substack{n\left|m \\ f_{\psi}\right| n}} b_{n, \bar{\psi}} \prod_{p \mid n}(1-\psi(p)),
$$


where $d=\left[K^{+}: \mathbb{Q}\right]$ and $c_{\chi}=\sum_{\tau \in C} \chi(\tau)$. In particular, if $D(0) \neq 0$ then the previous formula can be simplified as follows

$$
D(x)=\left(1-\frac{2 x}{d} \sum_{\chi \in X^{-}} \frac{\left|c_{\chi}\right|^{2}}{B_{1, \chi}\left(\sum_{n \mid m} b_{n, \bar{\chi}}\right) \prod_{p \mid n}(1-\chi(p))}\right) D(0) .
$$

Proof. Since

$$
(\chi(\tau))_{\substack{\chi \in X^{-} \\ \tau \in C}}(\bar{\psi}(\tau))_{\substack{\tau \in C \\ \psi \in X^{-}}}=\operatorname{diag}(d)_{\chi \in X^{-}}
$$

we have

$$
\operatorname{det}(\chi(\tau))_{\substack{\chi \in X^{-} \\ \tau \in C}} \cdot \operatorname{det}(\bar{\psi}(\tau))_{\substack{\tau \in C \\ \psi \in X^{-}}}=d^{d} .
$$

Therefore $D(x)=d^{-d} \operatorname{det} M$, where $^{3}$

$$
M=(\chi(\sigma))_{\substack{\chi \in X^{-} \\ \sigma \in C}} \cdot\left(x+t_{\sigma \tau^{-1}}\right)_{\sigma, \tau \in C} \cdot(\bar{\psi}(\tau))_{\substack{\tau \in C \\ \psi \in X^{-}}} .
$$

Because $t_{j \sigma}=-t_{\sigma}$ and $\chi$ is odd we obtain

$$
\begin{aligned}
\sum_{\sigma \in C} \sum_{\tau \in C} \chi(\sigma)\left(x+t_{\sigma \tau^{-1}}\right) \bar{\psi}(\tau) & =x c_{\chi} c_{\bar{\psi}}+\frac{1}{2} \sum_{\tau \in C} \chi(\tau) \bar{\psi}(\tau) \sum_{\sigma \in G} t_{\sigma \tau^{-1}} \chi\left(\sigma \tau^{-1}\right) \\
& =x c_{\chi} c_{\bar{\psi}}+\delta_{\chi, \psi} s_{\chi}
\end{aligned}
$$

where

$$
s_{\chi}=\frac{d}{2} \sum_{\sigma \in G} t_{\sigma} \chi(\sigma),
$$

and $\delta_{\chi, \psi}=1$ if $\chi=\psi$ and 0 otherwise. Let $X_{0}^{-}=\left\{\chi \in X^{-} ; c_{\chi} \neq 0\right\}$. Then

$\operatorname{det} M=\operatorname{det}\left(x c_{\chi} c_{\bar{\psi}}+\delta_{\chi, \psi} s_{\chi}\right)_{\chi, \psi \in X^{-}}$

$$
=\left(\prod_{\chi \in X^{-} \backslash X_{0}^{-}} s_{\chi}\right)\left(\prod_{\chi \in X_{0}^{-}}\left|c_{\chi}\right|^{2}\right) \operatorname{det}\left(x+\delta_{\chi, \psi} \frac{s_{\chi}}{\left|c_{\chi}\right|^{2}}\right)_{\chi, \psi \in X_{0}^{-}}
$$

and Lemma 5 gives

$$
\operatorname{det} M=\left(\prod_{\chi \in X^{-}} s_{\chi}\right)+x \sum_{\chi \in X^{-}}\left|c_{\chi}\right|^{2} \prod_{\substack{\psi \in X^{-} \\ \psi \neq \chi}} s_{\psi}
$$

\footnotetext{
${ }^{3}$ Let us mention that this method of computation was used by Wang in [29].
} 
For $\theta=\sum_{\sigma \in G} t_{\sigma} \sigma^{-1}$ we have

$$
\frac{s_{\chi}}{d}=\frac{1}{2} \rho_{K}^{\bar{\chi}}(\theta)=-\frac{1}{2} B_{1, \chi} \sum_{\substack{n\left|m \\ f_{\chi}\right| n}} b_{n, \bar{\chi}} \prod_{p \mid n}(1-\chi(p))
$$

(see the proof of Theorem 1) and the proposition follows.

Remark. It is easy to see that Proposition 1 is a generalization of Okada's result (3): for an odd prime $p$ take $K=K_{p}$ and $a_{p, \sigma}=-p$ if $\sigma=(1, K)$ and 0 otherwise. The following proposition is a generalization of Okada's Proposition (see [19]).

Proposition 2. If there is $\alpha=\sum_{\sigma \in G} n_{\sigma} \sigma^{-1} \in \mathbb{Q}[G]$ such that

$$
\frac{1-j}{2} \sum_{\sigma \in C} \sigma^{-1}=\alpha \theta
$$

then $D(x)=(1+2 n x) D(0)$, where

$$
n=\sum_{\sigma \in C} n_{\sigma}-\sum_{\sigma \in C} n_{j \sigma} .
$$

Proof. For $c_{\chi}$ defined in Proposition 1 we have

$$
c_{\chi}=\sum_{\sigma \in C} \chi(\sigma)=\rho_{K}^{\bar{\chi}}(\alpha \theta)=\rho_{K}^{\bar{\chi}}(\alpha) \rho_{K}^{\bar{\chi}}(\theta)
$$

and (19) gives

$$
\frac{\left|c_{\chi}\right|^{2}}{B_{1, \chi}}=-c_{\bar{\chi}} \rho_{K}^{\bar{\chi}}(\alpha) \sum_{\substack{n\left|m \\ f_{\chi}\right| n}} b_{n, \bar{\chi}} \prod_{p \mid n}(1-\chi(p)) .
$$

Proposition 1 and Theorem 1 imply

$$
D(x)-D(0)=\frac{2 x}{d} D(0) \sum_{\chi \in X^{-}} c_{\bar{\chi}} \rho_{K}^{\bar{\chi}}(\alpha) .
$$

We have

$$
\begin{aligned}
\sum_{\chi \in X^{-}} c_{\bar{\chi}} \rho_{K}^{\bar{\chi}}(\alpha) & =\sum_{\chi \in X^{-}} \sum_{\tau \in C} \bar{\chi}(\tau) \sum_{\sigma \in G} n_{\sigma} \chi(\sigma) \\
& =\sum_{\sigma \in G} n_{\sigma} \sum_{\tau \in C} \sum_{\chi \in X^{-}} \chi\left(\sigma \tau^{-1}\right) \\
& =\sum_{\sigma \in C} n_{\sigma} d+\sum_{\sigma \in G \backslash C} n_{\sigma}(-d),
\end{aligned}
$$


because

$$
\sum_{\chi \in X^{-}} \chi\left(\sigma \tau^{-1}\right)= \begin{cases}d & \text { if } \sigma=\tau \\ -d & \text { if } \sigma=j \tau \\ 0 & \text { otherwise. }\end{cases}
$$

The proposition follows.

EXAMPLE 5 . Let $m>1$ be an integer satisfying $m \not \equiv 2(\bmod 4)$. For any integers $a, b$ relatively prime to $m$ let $R_{m}\left(a b^{-1}\right)$ means the integer determined by $R_{m}\left(a b^{-1}\right) b \equiv a(\bmod m)$ and $0<R_{m}\left(a b^{-1}\right) \leq m$. Let $C$ be the set of all positive integers less than $m / 2$ which are relatively prime to $m$. Put $K=K_{m}$ and

$$
a_{n, \sigma}= \begin{cases}-m & \text { if } n=m \text { and } \sigma=(1, K) \\ 0 & \text { otherwise. }\end{cases}
$$

Then $b_{m, \chi}=-m$ and $b_{n, \chi}=0$ if $n \neq m$. Hence $t_{(s, K)}=m\left\langle\frac{s}{m}\right\rangle-\frac{m}{2}$ for any integer $s$ and $\theta=-m \frac{1-j}{2} \theta_{m}(1)$. For

$$
D(x)=\operatorname{det}\left(x+t_{(a, K)(b, K)^{-1}}\right)_{a, b \in C},
$$

Theorem 1 gives

$$
\begin{aligned}
D(0) & =\frac{h_{K}^{-}}{Q_{K} w_{K}} \prod_{\chi \in X^{-}}(-m) \prod_{p \mid m}(1-\bar{\chi}(p)) \\
& =\frac{h_{K}^{-}}{Q_{K} w_{K}}(-m)^{\left[K^{+}: \mathbb{Q}\right]} q_{K} .
\end{aligned}
$$

We want to compute

$$
\operatorname{det}\left(R_{m}\left(a b^{-1}\right)\right)_{a, b \in C}=D\left(\frac{m}{2}\right) .
$$

At first let us suppose that $m$ is even. Then

$$
\begin{aligned}
\frac{1-j}{2} \sum_{a \in C}(a, K)^{-1} & =\sum_{t \bmod ^{\times}}\left(\left\langle\frac{t}{m}+\frac{1}{2}\right\rangle-\left\langle\frac{t}{m}\right\rangle\right)(t, K)^{-1} \\
& =\theta_{m}\left(-1-\frac{m}{2}\right)-\theta_{m}(-1) \\
& =\left(-\left(1+\frac{m}{2}, K\right)+(1, K)\right) \frac{1-j}{2} \theta_{m}(1) \\
& =\left(\frac{1}{m}\left(1+\frac{m}{2}, K\right)^{-1}-\frac{1}{m}(1, K)^{-1}\right) \theta
\end{aligned}
$$


so Proposition 2 gives $n=-2 / m$ and $D(m / 2)=-D(0)$ in this case.

We shall suppose $m$ is odd now. Then

$$
\begin{aligned}
\frac{1-j}{2} \sum_{a \in C}(a, K)^{-1} & =-\frac{1-j}{2} \sum_{\substack{1 \leq a<m \\
(a, m)=1}}\left(\frac{2 a}{m}-\left\langle\frac{2 a}{m}\right\rangle\right)(a, K)^{-1} \\
& =-\frac{1-j}{2}\left(2 \theta_{m}(-1)-\theta_{m}(-2)\right) \\
& =(2(1, K)-(2, K)) \frac{1-j}{2} \theta_{m}(1) \\
& =\left(-\frac{2}{m}(1, K)^{-1}+\frac{1}{m}\left(\frac{m+1}{2}, K\right)^{-1}\right) \theta
\end{aligned}
$$

so Proposition 2 gives $n=-3 / m$ and $D(m / 2)=-2 D(0)$ in this case.

Putting both cases together we have obtained $D(m / 2)=-\frac{w_{K}}{m} D(0)$, so

$$
\begin{aligned}
\operatorname{det}\left(R_{m}\left(a b^{-1}\right)\right)_{a, b \in C} & =-\frac{w_{K}}{m} \frac{h_{K}^{-}}{Q_{K} w_{K}}(-m)^{\left[K^{+}: \mathbb{Q}\right]} q_{K} \\
& =(-m)^{\left[K^{+}: \mathbb{Q}\right]-1} \frac{q_{K}}{Q_{K}} \cdot h_{K}^{-},
\end{aligned}
$$

which gives (2).

\section{$\S 6$. Tsumura's formula}

This section is devoted to the comparison of the formulae derived independently by Tsumura and Hirabayashi. We shall show that the matrices in these formulae differ only by a permutation of rows, if we take a special case of Hirabayashi's formula, namely $b$ relatively prime to the conductor of the considered field (see (12) and (13)).

Let $K$ be an imaginary abelian field and let $l>1$ be an integer relatively prime to the conductor $m$ of $K$. For any integer $c$, Tsumura defines the rational number (see [28])

$$
A_{m}(c, l)=\sum_{\substack{\zeta^{l}=1 \\ \zeta \neq 1}} \frac{\zeta^{m-c}}{1-\zeta^{m}} .
$$

Tsumura's matrix $\Delta(K, l)$ can be defined as $\Delta(K, l)=\left(\delta_{\sigma \tau^{-1}}\right)_{\sigma, \tau \in C}$, where $C$ is a system of representatives of $\operatorname{Gal}(K / \mathbb{Q}) / \operatorname{Gal}\left(K / K^{+}\right)$and

$$
\delta_{\sigma}=2 \sum_{\substack{a \bmod ^{\times} m \\ 0<a<m \\(a, K)=\sigma}} A_{m}(a, l)
$$


for any $\sigma \in \operatorname{Gal}(K / \mathbb{Q})$. Tsumura proved

$$
\operatorname{det} \Delta(K, l)=(-2)^{\left[K^{+}: \mathbb{Q}\right]} \cdot \frac{h_{K}^{-}}{Q_{K} w_{K}} \cdot q_{K} \prod_{\chi \in X^{-}}(l \chi(l)-1) .
$$

LEMMA 6 . Let $k$ be an integer satisfying $k l \equiv 1(\bmod m)$. Then for any integer $c$ such that $0 \leq c<m$ we have

$$
A_{m}(c, l)=\left[l\left\langle\frac{k c}{m}\right\rangle\right]-\frac{l-1}{2} .
$$

Proof. We can suppose $k<0$. Let $n=(1-k l) / m$. The positive integer $n$ then satisfies $n m \equiv 1(\bmod l)$ and we have

$$
A_{m}(c, l)=\sum_{\substack{\zeta^{l}=1 \\ \zeta \neq 1}} \frac{\zeta^{-1+n c}}{1-\zeta^{-1}}=-\sum_{\substack{\zeta^{l}=1 \\ \zeta \neq 1}} \frac{\zeta^{n c}}{1-\zeta}=\sum_{\substack{\zeta^{l}=1 \\ \zeta \neq 1}} \frac{1-\zeta^{n c}}{1-\zeta}-\sum_{\substack{\zeta^{l}=1 \\ \zeta \neq 1}} \frac{1}{1-\zeta}
$$

But

$$
2 \sum_{\substack{\zeta^{l}=1 \\ \zeta \neq 1}} \frac{1}{1-\zeta}=\sum_{\substack{\zeta^{l}=1 \\ \zeta \neq 1}}\left(\frac{1}{1-\zeta}+\frac{1}{1-\zeta^{-1}}\right)=l-1
$$

and

$$
\begin{aligned}
\sum_{\substack{\zeta^{l}=1 \\
\zeta \neq 1}} \frac{1-\zeta^{n c}}{1-\zeta} & =\sum_{\substack{\zeta^{l}=1 \\
\zeta \neq 1}} \sum_{i=0}^{n c-1} \zeta^{i}=-n c+\sum_{\zeta^{l}=1} \sum_{i=0}^{n c-1} \zeta^{i} \\
& =-n c+l\left(\left[\frac{n c-1}{l}\right]+1\right)=l-1-l\left\langle\frac{n c-1}{l}\right\rangle
\end{aligned}
$$

The lemma follows if we use the identity

$$
\left[l\left\langle\frac{k c}{m}\right\rangle\right]=l-1-l\left\langle\frac{n c-1}{l}\right\rangle,
$$

which can be easily proved. Indeed, on both sides of this identity there are non-negative integers less than $l$ satisfying

$$
\begin{aligned}
{\left[l\left\langle\frac{k c}{m}\right\rangle\right] } & \equiv\left[\frac{l k c}{m}\right]=\left[\frac{c}{m}\right]-n c=-n c=-1-(n c-1) \\
& \equiv l-1-l\left\langle\frac{n c-1}{l}\right\rangle \quad(\bmod l)
\end{aligned}
$$


COROLlary 1. Tsumura's formula (20) can be derived from Hirabayashi's one (13).

Proof. Due to Lemma 6 we have $\delta_{\sigma}=2 t_{(k, K) \sigma}$ for any $\sigma \in \operatorname{Gal}(K / \mathbb{Q})$, where $t_{\sigma}$ is defined by (12) (take $b=l$ ). Therefore

$$
\Delta(K, l)=\left(\delta_{\rho \tau^{-1}}\right)_{\rho, \tau \in C}=\left(2 t_{(k, K) \rho \tau^{-1}}\right)_{\rho, \tau \in C}=\left(p_{\rho \sigma}\right)_{\rho, \sigma \in C}\left(2 t_{\sigma \tau^{-1}}\right)_{\sigma, \tau \in C}
$$

where $\left(p_{\rho \sigma}\right)_{\rho, \sigma \in C}$ is a permutation matrix given by

$$
p_{\rho \sigma}= \begin{cases}1 & \text { if }(k, K) \rho=\sigma \\ -1 & \text { if }(-k, K) \rho=\sigma \\ 0 & \text { otherwise }\end{cases}
$$

We need the sign of the determinant of $\left(p_{\rho \sigma}\right)_{\rho, \sigma \in C}$. Multiplying by the character matrix, which is well known to be non-singular, gives

$$
\left(p_{\rho \sigma}\right)_{\rho, \sigma \in C}(\chi(\sigma))_{\sigma \in C, \chi \in X^{-}}=(\chi(\rho))_{\rho \in C, \chi \in X^{-}} \operatorname{diag}(\chi(k))_{\chi \in X^{-}}
$$

and so $\operatorname{det}\left(p_{\rho \sigma}\right)_{\rho, \sigma \in C}=\prod_{\chi \in X^{-}} \chi(k)$. Then (13) gives

$$
\operatorname{det} \Delta(K, l)=(-2)^{\left[K^{+}: \mathbb{Q}\right]} \cdot c_{K}(b) \cdot \frac{h_{K}^{-}}{Q_{K} w_{K}} \cdot \prod_{\chi \in X^{-}} \chi(k),
$$

and (20) follows by a straightforward calculation.

Acknowledgements. I am grateful to Claude Levesque for many remarks which improved this paper.

\section{REFERENCES}

[1] T. Agoh and L. Skula, Kummer type congruences and Stickelberger subideals, Acta Arith., 75 (1996), 235-250.

[2] L. Carlitz and F. R. Olson, Maillet's determinant, Proc. Amer. Math. Soc., 6 (1955), 265-269.

[3] K. Dohmae, Demjanenko's matrix for imaginary abelian number fields of odd conductors, Proc. Japan Acad., 70 (1994), 292-294.

[4] A. Endô, The relative class numbers of certain imaginary abelian number fields and determinants, J. Number Theory, 34 (1990), 13-20.

[5] P. Fuchs, Maillet's determinant and a certain basis of the Stickelberger ideal, Tatra Mount. Math. Publ., 11 (1997), 121-128. 
[6] K. Girstmair, An index formula for the relative class number of an abelian number field, J. Number Theory, 32 (1989), 100-110.

[7] K. Girstmair, On the cosets of the 2q-th power group in the unit group modulo $p$, Abh. Math. Sem. Univ. Hamburg, 62 (1992), 217-232.

[8] K. Girstmair, The relative class number of imaginary cyclic fields of degrees 4, 6, 8, and 10, Math. of Comp., 61 (1993), 881-887.

[9] H. Hasse, Über die Klassenzahl abelscher Zahlkörper, Akademie-Verlag, Berlin, 1952.

[10] F. Hazama, Demjanenko matrix, class number, and Hodge group, J. Number Theory, 34 (1990), 174-177.

[11] F. Hazama, Hodge cycles on the jacobian variety of the Catalan curve, Compositio Math., 107 (1997), 339-353.

[12] M. Hirabayashi, A relative class number formula for an imaginary abelian field by means of Demjanenko matrix, Proceedings of Conference on Analytic and Elementary Number Theory, Viena 1996 (W. G. Nowak and J. Schoißengeier, eds.), pp. 81-91.

[13] M. Hirabayashi, A generalization of Maillet and Demjanenko determinants, preprint (1996).

[14] S. Kanemitsu and T. Kuzumaki, On a generalization of the Maillet determinant, preprint (1997).

[15] J. Kühnová, Maillet's determinant $D_{p^{n+1}}$, Arch. Math. (Brno), 15 (1979), 209-212.

[16] R. Kučera, On the Stickelberger ideal and circular units of a compositum of quadratic fields, J. Number Theory, 56 (1996), 139-166.

[17] G. Lettl, Stickelberger elements and cotangent numbers, Expositiones Math., 10 (1992), 171-182.

[18] T. Metsänkylä, Bemerkungen über den ersten Faktor der Klassenzahl des Kreiskörpers, Ann. Univ. Turku. Ser. A I, 105 (1967), 15 pp.

[19] S. Okada, Generalized Maillet determinant, Nagoya Math. J., 94 (1984), 165-170.

[20] J. W. Sands and W. Schwarz, A Demjanenko matrix for abelian fields of prime power conductor, J. Number Theory, 52 (1995), 85-97.

[21] W. Schwarz, Demjanenko matrix and 2-divisibility of class numbers, Arch. Math. (Basel), 60 (1993), 154-156.

[22] W. Sinnott, On the Stickelberger ideal and the circular units of a cyclotomic field, Ann. of Math., 108 (1978), 107-134.

[23] W. Sinnott, On the Stickelberger ideal and the circular units of an abelian field, Inv. Math., 62 (1980), 181-234.

[24] L. Skula, Another proof of Iwasawa's class number formula, Acta Arith., 39 (1981), $1-6$.

[25] L. Skula, The Stickelberger ideal and the Demjanenko matrix, Algebraic Cycles and Related Topics (F. Hazama, ed.), World Scientific, Singapore (1995), pp. 69-76.

[26] L. Skula, On a special ideal contained in the Stickelberger ideal, J. Number Theory, 58 (1996), 173-195.

[27] K. Tateyama, Maillet's determinant, Sci. Papers Coll. Gen. Edu. Univ. Tokyo, 32 (1982), 97-100. 
[28] H. Tsumura, On Demjanenko's matrix and Maillet's determinant for imaginary abelian number fields, J. Number Theory, 60 (1996), 70-79.

[29] K. Wang, On Maillet determinant, J. Number Theory, 18 (1984), 306-312.

[30] L. C. Washington, Introduction to cyclotomic fields, Springer-Verlag, New York, 1982.

Department of Mathematics

Faculty of Science

Masaryk University

Janáčkovo nám. $2 a$

662 95 Brno

Czech Republic

kucera@math.muni.cz 